\title{
Color Triplet Diquarks at the LHC
}

\author{
Ilia Gogoladzed1, Yukihiro Mimura ${ }^{a}$, Nobuchika Okada ${ }^{b}$ and Qaisar Shafi ${ }^{a}$ \\ ${ }^{a}$ Bartol Research Institute, Department of Physics and Astronomy, \\ University of Delaware, Newark, DE 19716, USA \\ ${ }^{b}$ Department of Physics and Astronomy, University of Alabama, Tuscaloosa, AL 35487, USA
}

\begin{abstract}
We consider a class of supersymmetric models containing baryon number violating processes such as observable $n-\bar{n}$ oscillations that are mediated by color triplet diquark fields. For plausible values of the diquark-quark couplings, the scalar diquark with mass between a few hundred $\mathrm{GeV}$ and one $\mathrm{TeV}$ or so can be produced in the $s$-channel at the LHC and detected through its decay into a top quark and a hadronic jet.
\end{abstract}

\footnotetext{
${ }^{1}$ On leave of absence from: Andronikashvili Institute of Physics, Tbilisi, Georgia.
} 


\section{Introduction}

Being a proton-proton $(p p)$ collider the LHC is an ideal hunting ground for new elementary particles which carry non-zero baryon number. Such particles often arise as vectorlike pairs in a large class of supersymmetric (SUSY) models, including some which are obtained from compactification of superstring theories [1, 2, 3].

The $\mathrm{TeV}$ scale vectorlike particles have various indirect phenomenological applications. They can help to substantially increase the mass of the lightest CP-even Higgs boson of minimal SUSY standard model (MSSM) 4, 5]. The new Higgs coupling to these particles can also modify the first order electroweak phase transition, and thereby make the scalar top quark mass bound milder in the electroweak baryogenesis scenario [6]. The leptonic vectorlike particles can generate sizable contribution to the tau and tau neutrino electric dipole moment which can be tested in future experiment [7].

The low scale vectorlike particle can also induce observable neutron-antineutron $(n-\bar{n})$ oscillations [8, 9], in which the so-called diquark couplings play a crucial role. The vectorlike field which couples to two anti-quarks have baryon number $2 / 3$ (namely twice the baryon number of quark), and thus we refer to such a colored vectorlike field as "diquark". The coupling between diquarks and a pair of quarks can make the creation and decay modes of the diquarks very interesting in view of collider physics [10]. If the diquark fields are pair produced at the LHC through QCD processes [11, 12], they decay into quarks via the diquark interaction. This interaction also plays a key role in the resonant production of the diquark field from $p p$ collision, and if the cross section is large enough to distinguish the peak from the standard model (SM) background, one can detect the diquark field and determine its mass [13].

There are two possibilities for the representation of the diquark fields: (1) color triplet, and (2) color sextet. The color sextet diquark fields can be embedded in the Higgs multiplet required to break the gauge symmetry $S U(4)_{c} \times S U(2)_{L} \times S U(2)_{R}$ [14] down to SM, and the mass of the diquark can remain around a $\mathrm{TeV}$ scale if there exists an accidental global symmetry in the Higgs potential [8]. Such sextet diquarks do not have a renormalizable coupling with leptons, and their existence at low energy is not problematic. The color triplet diquarks are also well motivated since they can be accommodated into matter representations in unified models [2]. However, they can couple to both quarks and leptons, and the most general couplings can cause serious problems with rapid nucleon decay if the diquark mass is around the $\mathrm{TeV}$ scale. This difficulty is remedied by employing a $U(1)$ symmetry to prohibit the couplings of the color triplets with leptons [9]. In this case, there are no dangerous nucleon decay operators, but the $U(1)$ symmetry can allow baryon number violating processes [9] like $n-\bar{n}$ oscillations.

In this paper, motivated by the physics of $n-\bar{n}$ oscillations, we study the resonant production 
and decay of the triplet diquarks at the LHC. The attractive feature of gauge coupling unification in MSSM is easily maintained [3] in the case of triplet diquarks accompanied by electroweak doublets. Clearly, the diquark couplings with the up and down quarks should be large enough to detect its resonant production. However, some of the couplings (especially for the first and second generations) are constrained by the experimental data on meson-antimeson $(K-\bar{K}, B-\bar{B}$ and $\left.D^{0}-\bar{D}^{0}\right)$ mixings, and thus we will first discuss the experimentally allowed parameter range of the diquark couplings. In the case of sextet diquark, $D^{0}-\bar{D}^{0}$ mixing is generated even at treelevel, while for the triplet diquarks, the mixings are generated by box or penguin diagrams, quite similar to the conventional analysis in MSSM. We will study whether one can see the resonant peak from the SM background in the phenomenologically allowed region of the diquark coupling. If the triplet diquarks couple with quarks for both first and third generations, one can observe resonant decay events for both hadronic dijet and single top quark plus one jet. Note that the triplet diquark can couple to both left- and right-handed quarks, while the sextet diquarks can couple only with the right-handed quarks. It is therefore important to observe top quark helicity to distinguish these two cases of diquark production from each other.

This paper is organized as follows: In section 2, we introduce following [9] a $U(1)$ symmetry which forbids rapid nucleon decay processes but allows $n-\bar{n}$ oscillations. In section 3 , the phenomenological constraints of the diquark couplings are considered. The diquark can couple with the quarks of all generations. In section 4, we study the collider phenomenology of the triplet diquarks. We first study the mass bounds on diquarks arising from the Tevatron results, and then discuss the resonant production and decays of the diquarks at the LHC. Section 5 summarizes our conclusions.

\section{$2 \quad$ A model with triplet diquark couplings}

We introduce the following vectorlike fields (more precisely chiral superfield) with masses in the few hundred GeV to TeV range:

$$
D:(\mathbf{3}, \mathbf{1})_{-\frac{1}{3}}+\bar{D}:(\overline{\mathbf{3}}, \mathbf{1})_{\frac{1}{3}}+L:(\mathbf{1}, \mathbf{2})_{-\frac{1}{2}}+\bar{L}:(\mathbf{1}, \mathbf{2})_{\frac{1}{2}} .
$$

Because $\bar{D}+L$ has the same particle content as the 5-dimensional $S U(5)$ multiplet, gauge coupling unification in MSSM is preserved. The vectorlike fields can couple with the quark and lepton fields as follows:

$$
D q q+D u^{c} e^{c}+\bar{D} q \ell+\bar{D} u^{c} d^{c}+L \ell e^{c}+L q d^{c}+\bar{L} q u^{c}
$$

Here we use the standard notation $\left(q, u^{c}, d^{c}, \ell, e^{c}\right)$ for the quarks and leptons. In general, the couplings in Eq.(2) contradict the current experimental data [15] unless the coupling coefficients 


\begin{tabular}{|c|c|c|c|c|c|c|c|c|c|c|c|c|}
\hline$q$ & $u^{c}$ & $d^{c}$ & $\ell$ & $e^{c}$ & $\nu^{c}$ & $H_{u}$ & $H_{d}$ & $D$ & $\bar{D}$ & $L$ & $\bar{L}$ & $S$ \\
\hline$\frac{n}{6}$ & $-\frac{n}{6}$ & $-\frac{n}{6}$ & $\frac{m}{2}$ & $-\frac{m}{2}$ & $-\frac{m}{2}$ & 0 & 0 & $-\frac{n}{3}-\frac{X_{D}}{2}$ & $\frac{n}{3}+\frac{X_{D}}{2}$ & $-\frac{n}{2}-\frac{X_{L}}{2}$ & $\frac{n}{2}+\frac{X_{L}}{2}$ & 1 \\
\hline
\end{tabular}

Table 1: Particle charge assignments under $(n B+m L) / 2$ symmetry. The numbers $n, m, X_{D}$ and $X_{L}$ are all integers.

are less than $O\left(10^{-13}\right)$. Indeed, the exchange of the scalar component of $D(\bar{D})$ chiral multiplet can generate the dimension-six proton decay operator $q q u^{c \dagger} e^{c \dagger}\left(q \ell u^{c \dagger} d^{c \dagger}\right)$, which leads to an unacceptable proton decay rate.

The low scale vectorlike multiplets $(\mathbf{5}+\overline{\mathbf{5}})$ can, in general, generate neutrino masses through loop effects if the following interactions are allowed:

$$
q \bar{D} H_{d}+L e^{c} H_{d}+\bar{D} q \ell+L \ell e^{c}
$$

where $H_{d}$ stands for the MSSM down-type Higgs field. In this case, one has to assume the couplings in Eq.(3) to be $O\left(10^{-4}\right)$ to obtain the correct neutrino masses. Since our goal in this paper is to investigate resonant production of diquark at the LHC, we desire to have at least $O(0.1)$ strength couplings of diquarks with the SM quarks.

Thus, it seems natural to forbid rapid proton decay and large neutrino masses by a symmetry. Clearly, if odd $R$-parity is assigned to the vectorlike fields, the couplings in Eq.(2) are all forbidden, which clearly is not our goal. It was shown in Ref. [9] that a $(n B+m L) / 2$ $U(1)$ symmetry, where $B$ and $L$ are the baryon and lepton numbers of the fields and $n$ and $m$ are integers, can allow us to retain the "diquark couplings", $D q q$ and $\bar{D} u^{c} d^{c}$, but forbid the "leptoquark couplings", $D u^{c} e^{c}$ and $\bar{D} q \ell$. As a consequence, the rapid nucleon decay operators are forbidden. If odd $R$-parities are assigned for $L+\bar{L}$ and even $R$-parity for $D+\bar{D}$, then all the terms in Eq.(3) can be forbidden and therefore neutrino masses will not be generated from the loop effects.

Let us describe in more detail how the $(n B+m L) / 2$ symmetry works. The charge assignments for quarks, leptons, Higgs and vectorlike fields under $(n B+m L) / 2$ symmetry are given in Table 1. The $U(1)$ symmetry is anomalous and the Green-Schwarz mechanism [16] is applied. The SM singlet field $S$ acquires a vacuum expectation value (VEV) close to the string scale $M_{s t}$ from the Fayet-Illiopoulos $D$-term potential. If $n+m$ is an odd integer, the $\Delta B=\Delta L= \pm 1$ operators (e.g., $q q q \ell, u^{c} d^{c} u^{c} e^{c}, q q u^{c \dagger} e^{c \dagger}$ ) are all forbidden, as long as no field with fractional charge of $(n B+m L) / 2$ symmetry acquires a VEV. The couplings of quarks and leptons with $D+\bar{D}$ fields are expressed as

$$
\left(\frac{S}{M_{s t}}\right)^{\frac{X_{D}}{2}} D q q+\left(\frac{S}{M_{s t}}\right)^{\frac{X_{D}}{2}} \bar{D} u^{c} d^{c}+\left(\frac{S}{M_{s t}}\right)^{\frac{x_{D}+n+m}{2}} D u^{c} e^{c}+\left(\frac{S}{M_{s t}}\right)^{-\frac{x_{D}+n+m}{2}} \bar{D} q \ell .
$$


If the exponents of $S$ are not integers, the respective couplings are not allowed. As one can see, the diquark couplings are allowed only if $n+m$ is an odd number and $X_{D}$ is even.

It is interesting to note that the $(n B+m L) / 2$ symmetry forbids rapid nucleon decay, but it can allow baryon number violating processes like the $\Delta B=2 n-\bar{n}$ oscillations. In fact, the appropriate operators responsible for $n-\bar{n}$ oscillations are effectively generated [9] if there are MSSM singlet fields $N:(\mathbf{1}, \mathbf{1})_{0}$, in addition to the diquark fields. The typical $n$ - $\bar{n}$ oscillation operators are $u^{c} d^{c} d^{c} u^{c} d^{c} d^{c}$ and $q q d^{c \dagger} q q d^{c \dagger}$, and the diquark couplings, $\bar{D} u^{c} d^{c}$ and $D q q$, play a crucial role in generating them at tree level. Through such couplings, the diquark can decay into pairs of up-type and down-type quarks and generate a hadronic dijet or a single top quark plus a hadronic jet.

In order to induce the $n-\bar{n}$ oscillation operators, one can also add a pair of vectorlike fields

$\bar{U}:(\overline{\mathbf{3}}, \mathbf{1})_{-2 / 3}+U:(\mathbf{3}, \mathbf{1})_{2 / 3}$, and a diquark coupling $\bar{U} d_{i}^{c} d_{j}^{c}$. Since the representations are included in $\mathbf{1 0}+\overline{\mathbf{1 0}}$ multiplets of $S U(5)$ symmetry, the MSSM gauge coupling unification can be maintained if the complete contents of $\mathbf{1 0}+\overline{\mathbf{1 0}}$ representations are introduced. Through the diquark coupling, the $\bar{U}$ diquark decay produces only hadronic dijet. Although at high energy hadron colliders the dijet signal from the resonant production of the diquarks seems to be overwhelmed by QCD background, the large luminosity at the Tevatron allows us to give a fairly severe lower bound on the diquark mass [17]. Depending on the couplings, the diquarks can be discovered at the LHC through the dijet events [18. Noting that the $D+\bar{D}$ diquarks can decay into single top quark plus a hadronic jet, we concentrate in this paper on collider phenomenology of these diquarks and their decays into top quarks. We find that this process involving the top quark gives a lower bound on the diquark mass which is more severe than that obtained from the dijet process. Furthermore, the final state top quarks are useful in identifying the baryon number of the diquark and a chiral structure of the coupling between the diquarks and quarks.

\section{Phenomenological constraint on the diquark couplings}

The magnitude of the coupling between the diquarks and quarks is clearly important for the production and decay modes of the diquark fields at the LHC, and we therefore first study the phenomenological constraints on these couplings. We define the diquark coupling constants as

$$
\frac{1}{2} \kappa_{i j} D q_{i} q_{j}+\bar{\kappa}_{i j} \bar{D} u_{i}^{c} d_{j}^{c}
$$

where $i, j$ are flavor indices. The most important constraint on the coupling constants and the vectorlike mass of $D, \bar{D}$ arise from the oblique corrections for the weak gauge bosons ( $S$ and 
$T$ parameters) [19, 20]. The mass and couplings of the diquarks are also constrained from the Tevatron experiment, which will be discussed in the next section.

In SUSY models, the diquark couplings can induce flavor non-universality in SUSY breaking squark mass matrices through the renormalization group ( $R G$ ) evolutions, even if universality is assumed at the unification scale. The flavor violation effects can generate processes via the flavor changing neutral current (FCNC) (e.g., meson-antimeson mixings and $b \rightarrow s(d) \gamma$ ) through loop diagrams involving SUSY particles. Therefore, generic $O(0.1)$ coupling coefficients for the first and second generations are disfavored. In addition to such indirect effects of FCNC, the diquark couplings can generate FCNC loop diagrams in which the diquark fields propagate.

We note that $D^{0}-\bar{D}^{0}$ meson mixing is generated even at the tree level if a sextet diquark field $\Delta:(6,1)_{\frac{4}{3}}$ and the coupling $\Delta u_{i}^{c} u_{j}^{c}$ are considered. However, in the case of triplet diquarks (including $U+\bar{U}$ ), all FCNC processes are generated at the loop level, and the essential features for finding the experimental bounds are similar to the MSSM case [21]. Thus the matrix elements of $\kappa \kappa^{\dagger}, \kappa \bar{\kappa}$, etc., are constrained for a given mass spectrum.

If the diquark coupling matrix elements are hierarchical, similar to the Yukawa couplings for quarks and leptons, the FCNC effects can be adequately suppressed. However, for the resonant production of diquarks at the LHC, we prefer a parameter region where the couplings for the first generation $\kappa_{11}$ (or $\kappa_{12}$ ) are of $O(0.1)$. We make the following simplifying ansatz for minimal flavor violation:

$$
\kappa_{i j}=\kappa \delta_{i j}, \quad \bar{\kappa}_{i j}=\bar{\kappa} \delta_{i j} .
$$

In this case, the RG evolutions do not generate any new sources of FCNC in the squark mass matrices. The new direct loop contributions via the diquark fields, however, are generated from the left-handed coupling $\kappa D q q$ because of the Cabibbo-Kobayashi-Maskawa (CKM) mixings. The contributions to FCNC processes under the assumption in Eq.(6) is similar to the chargino loop diagram (except for the charge and couplings), and thus the amount of the contribution is not significant compared to that of the SM when flavor universality of the squark squared masses is assumed at a unification scale. Therefore, for minimal flavor violation, the experimental constraint is satisfied as long as the diquarks are heavier than about $300 \mathrm{GeV}$, and $\kappa \leq 1$. Actually, if the diquark coupling is more than $O(0.1)$, the diquark should be heavier than about $300 \mathrm{GeV}$ from the Tevatron result, as we will see in next section.

Any deviation from Eq.(6) is severely constrained from $K-\bar{K}$ mixings, especially for $\kappa_{11}-\kappa_{22}$, $\kappa_{12}$ and $\bar{\kappa}_{12} \lesssim O\left(10^{-3}\right)-O\left(10^{-4}\right)$. The $B_{d, s} \bar{B}_{d, s}$ mixings and $b \rightarrow s(d) \gamma$ decay give $\kappa_{23}, \bar{\kappa}_{23}$ $\lesssim O\left(10^{-2}\right)$, and $\kappa_{13}, \bar{\kappa}_{13} \lesssim O\left(10^{-3}\right)$. Such small contributions in the flavor off-diagonal elements are not crucial for the collider studies, and so will be disregarded in what follows.

Through the RG running, the squark masses become smaller at low energy, for a given 
boundary condition, as the diquark couplings get larger. Under the assumption in Eq.(6), the SUSY breaking squared scalar masses of diquarks become negative when the coupling is $O(1)$ at low energy due to the fact that the wave function renormalizations of the diquark fields are proportional to $\operatorname{Tr}\left(\kappa \kappa^{\dagger}\right)$ and $\operatorname{Tr}\left(\bar{\kappa} \bar{\kappa}^{\dagger}\right)$. To avoid color and charge symmetry breaking, an appropriate size vectorlike mass of the diquark them needs to be added, in which case the fermionic partners of the diquarks are heavier than the scalar diquarks. If the SUSY breaking diquark squared masses are negative, the squarks do not become tachyonic even if the diquark coupling is large. If the diquark couplings are about 0.3 at low energy, the squared scalar mass will not become negative due to the gluino loop contribution and the fermionic diquark is lighter than the scalar diquark.

\section{Collider phenomenology}

Since the scalar diquarks ( $D$ and $\bar{D}$ ) couple with a pair of quarks, they can be produced in the $s$-channel at the Tevatron and the LHC through annihilation of a pair of quarks. As a signature of scalar diquark productions at hadron colliders, we concentrate on its decay channel which includes a single top or anti-top quark in the final state. Because of its mass, the top quark decays electroweakly before hadronizing. Due to this characteristic feature not shared by the other quarks, the top quark can be an ideal tool [22] to probe new physics beyond the standard model [23].

We consider here the scalar diquark $(D)$ production and the analysis for $\bar{D}$ is basically the same, except for the chirality of the initial and final quarks. The fundamental processes are $u d \rightarrow D \rightarrow t b\left(\bar{u} \bar{d} \rightarrow D^{\dagger} \rightarrow \bar{t} \bar{b}\right.$ for the scalar anti-diquark production). The cross section at the parton level is given by

$$
\frac{d \sigma(u d \rightarrow D \rightarrow t b)}{d \cos \theta}=\frac{|\kappa|^{4}}{48 \pi} \frac{\left(\hat{s}-m_{t}^{2}\right)^{2}}{\left(\hat{s}-M_{D}^{2}\right)^{2}+M_{D}^{2} \Gamma_{\text {tot }}^{2}} .
$$

Here, we have neglected all quark masses except for top quark mass $\left(m_{t}=173.1 \mathrm{GeV}[24]\right), \theta$ is the scattering angle, and the total decay width $\Gamma_{\text {tot }}$ of the scalar diquark is the sum of its partial decay widths,

$$
\begin{aligned}
\Gamma(D \rightarrow u d, c s) & =\frac{1}{8 \pi}|\kappa|^{2} M_{D} \\
\Gamma(D \rightarrow t b) & =\frac{1}{8 \pi}|\kappa|^{2} M_{D}\left(1-\frac{m_{t}^{2}}{M_{D}^{2}}\right)^{2}
\end{aligned}
$$

Note that the cross section is independent of the scattering angle because the diquark is a scalar. 
At the Tevatron, the total production cross section of a quark pair $(t b)$ through the scalar diquark in the $s$-channel is given by

$$
\begin{aligned}
\sigma(p \bar{p} \rightarrow t b+X) & =\int d x_{1} \int d x_{2} \int d \cos \theta\left[f_{u}\left(x_{1}, Q^{2}\right) f_{\bar{d}}\left(x_{2}, Q^{2}\right)+f_{\bar{u}}\left(x_{1}, Q^{2}\right) f_{d}\left(x_{2}, Q^{2}\right)\right] \\
& \times \frac{d \sigma\left(u d \rightarrow D \rightarrow t b ; \hat{s}=x_{1} x_{2} E_{\mathrm{CMS}}^{2}\right)}{d \cos \theta}
\end{aligned}
$$

where $f_{q}$ denotes the parton distribution function for a quark $q$ in a proton, and $E_{\mathrm{CMS}}$ is the collider energy. Note that one parton distribution function is for the up (down) quark and the other is for the sea down (up) quark. At the Tevatron the production cross sections of $D$ and $D^{\dagger}$ are the same, reflecting the fact that the total baryon number of the initial state $p \bar{p}$ is zero.

At the LHC, the total $t b$ production cross section is given by

$$
\begin{aligned}
\sigma(p p \rightarrow t b+X) & =\int d x_{1} \int d x_{2} \int d \cos \theta \\
& \times 2 f_{u}\left(x_{1}, Q^{2}\right) f_{d}\left(x_{2}, Q^{2}\right) \frac{d \sigma\left(u d \rightarrow D \rightarrow t b ; \hat{s}=x_{1} x_{2} E_{\mathrm{CMS}}^{2}\right)}{d \cos \theta} .
\end{aligned}
$$

Here, both parton distribution functions are for the valence quarks, corresponding to a protonproton system at the LHC. The total production cross section of $\bar{t} \bar{b}$ through the scalar antidiquark production in the $s$-channel is obtained by replacing with the appropriate parton distribution function for anti-quarks. The initial $p p$ state has a positive baryon number, so that the production cross section at the LHC of $D$ is much larger than the one for $D^{\dagger}$. The dependence of the cross section on the final state invariant mass $M_{\text {inv }}$ is given by

$$
\frac{d \sigma(p p \rightarrow t b+X)}{d M_{\mathrm{inv}}}=\int_{\frac{M_{\mathrm{inv}}^{2}}{E_{\mathrm{CMS}}^{2}}}^{1} d x \frac{4 M_{\mathrm{inv}}}{x E_{\mathrm{CMS}}^{2}} f_{u}\left(x, Q^{2}\right) f_{d}\left(\frac{M_{\mathrm{inv}}^{2}}{x E_{\mathrm{CMS}}^{2}}, Q^{2}\right) \sigma\left(u d \rightarrow D \rightarrow t b ; \hat{s}=M_{\mathrm{inv}}^{2}\right) .(
$$

Let us first examine the lower bound on the scalar diquark mass from the Tevatron. The production of single top quark at the Tevatron at $\sqrt{s}=1.96 \mathrm{TeV}$ has been observed, and the measured cross section is consistent with the standard model prediction [25]. We consider the cross section [26],

$$
\sigma(p \bar{p} \rightarrow t b+X)=1.05 \pm 0.81 \mathrm{pb}
$$

from $s$-channel $\mathrm{W}$ boson, and use it as a constraint on $t b$ production cross section through the scalar diquark in the $s$-channel. Any possible new physics should be in the uncertainty range of this cross section, and so we take the bound to be

$$
\sigma(p \bar{p} \rightarrow D \rightarrow t b) \lesssim 0.81 \mathrm{pb}
$$




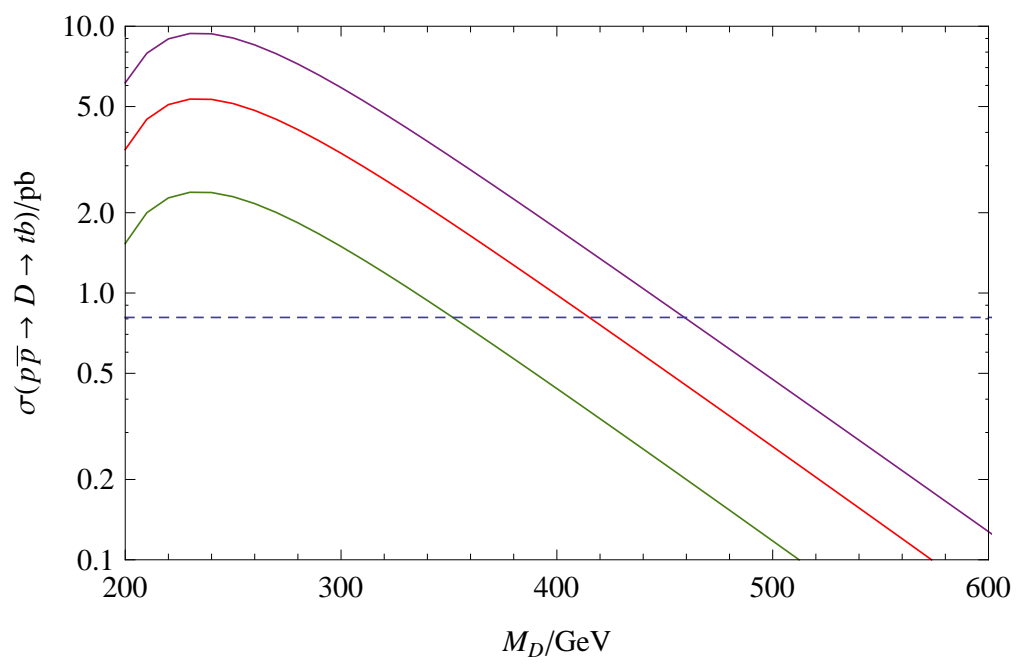

Figure 1: The cross section of $t b$ production at the Tevatron with $E_{\mathrm{CMS}}=1.96 \mathrm{TeV}$ mediated by the scalar diquark in the $s$-channel. Solid lines correspond to $\kappa=0.2,0.3,0.4$ from bottom to top. The horizontal dotted line shows the Tevatron bound.

In our numerical analysis, we employ CTEQ5M [27] for the parton distribution functions with the factorization scale $Q=m_{t}=173.1 \mathrm{GeV}$. Figure 1 shows the total cross section of $t b$ production as a function of the scalar diquark mass, with $E_{\mathrm{CMS}}=1.96 \mathrm{TeV}$. The lower bound on the diquark mass is found to be 352,415 , and $459 \mathrm{GeV}$, for $\kappa=0.2,0.3,0.4$, respectively. For these values of $\kappa$, we also evaluate the dijet production cross section via the resonant diquark production and find that the signal cross section is below the observed upper limit [17] and, hence, there no lower bound is obtained. We also find that for a sufficiently large value of $\kappa$, the lower bound on the diquark mass obtained by the observed cross section of single top quark production is always more severe than that from the dijet production cross section.

Next we investigate the scalar diquark and anti-diquark productions at the LHC with $E_{\mathrm{CMS}}=14 \mathrm{TeV}$. The differential cross sections for each process for $\kappa=0.3$ and with $M_{D}=600$ $\mathrm{GeV}$ and $1 \mathrm{TeV}$ are depicted in Figure 2. Here, we compare the single top quark production via the scalar diquark with the $t \bar{t}$ production cross section in the standard model. At the LHC, the main background for the single top quark production is $t \bar{t}$ production [28], and it is easy to misidentify $t \bar{t}$ events as single top events. So, for a conservative analysis, we compare our signal to $t \bar{t}$ production. The single top production cross section is smaller than the $t \bar{t}$ production cross section at the LHC by factor of about 7 .

We can see that the peak cross sections for $t b$ production exceeds the standard model cross section, while the $\bar{t} \bar{b}$ cross section by comparison is lower (however, the $\bar{t} \bar{b}$ cross section deviates sizably from the standard model cross section). This discrepancy between the production cross sections of scalar diquark and anti-diquark at the LHC is direct evidence for non-zero baryon 


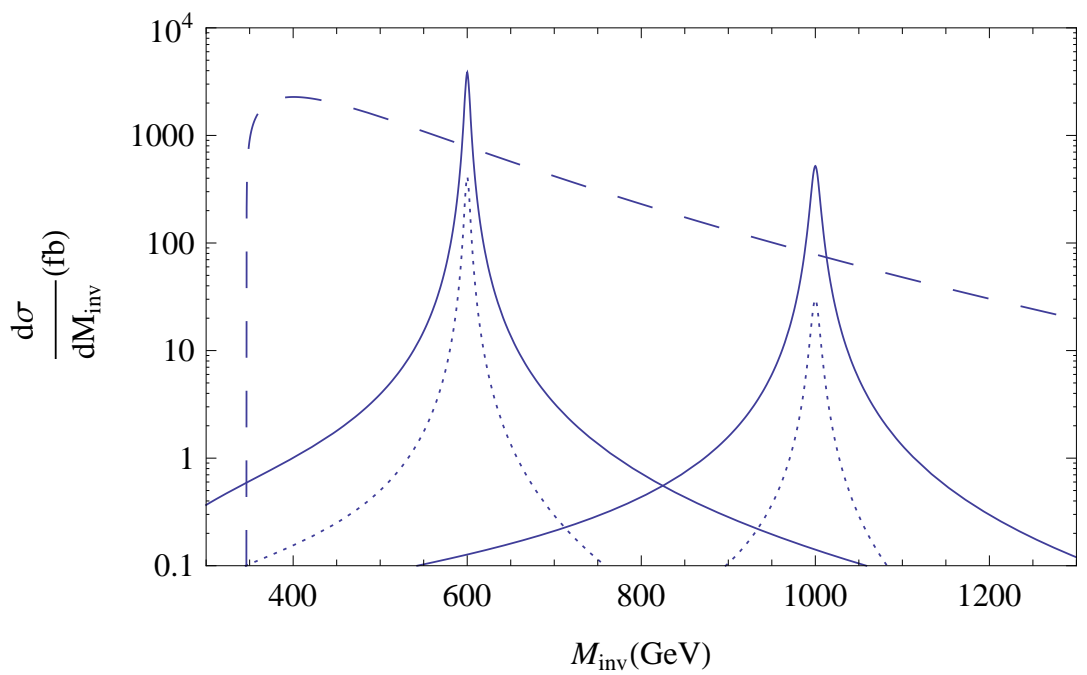

Figure 2: The differential cross sections for $t b$ (solid line), $\bar{t} \bar{b}$ (dotted line) versus the invariant mass of the final states. The left peak corresponds to $M_{D}=600 \mathrm{GeV}$ and the right one to $M_{D}=1 \mathrm{TeV}$. The dashed line is the standard model $t \bar{t}$ background. Here $\kappa=0.3$

number of the scalar diquark. Note that one can distinguish the top quark from the antitop quark through their semi-leptonic decays. Counting the number of top quark events and anti-top quark events would reveal a non-zero baryon number for the scalar diquark.

The result for the production of the scalar diquark $\bar{D}$ is the same when we take $\kappa=\bar{\kappa}$ and replace $D^{\dagger}$ with $\bar{D}$. However, there is a crucial difference in the spin polarization of the final state top (anti-top) quark. This is because while $D$ couples with the left-handed top quark, $\bar{D}^{\dagger}$ couples with the right-handed top quark. Since the top quark decays before hadronizing, the information of the top quark spin polarization is directly transferred to its decay products and results in significant angular correlations between the top quark polarization axis and the direction of motion of the decay products [29]. It has been shown that measuring the top spin correlations can increase the sensitivity for detecting a new particle at the Tevatron 30] and the LHC [31]. For the scalar diquark production, we can distinguish between $D$ and $\bar{D}^{\dagger}$ productions by measuring the polarization of top quarks from their decays. It is interesting that only the right-handed top quark is produced by the scalar diquark $\bar{D}^{\dagger}$ decay, while the single top quark produced through the standard model process is purely left-handed.

We have discussed the scalar diquark production in the $s$-channel, where the production cross section is controlled by the diquark coupling. Because they carry color, diquarks can be pair produced through QCD processes, and this production is independent of the diquark coupling. For the sextet diquark, the pair production at the LHC has been investigated in detail [11]. Although the analysis there focused on $t t \overline{t t}$ final state, a similar analysis here for the pair production of triplet scalar diquarks is applicable. The total pair production cross 
section of the triplet diquark is about an order of magnitude smaller than the sextet diquark case because of the difference of the $S U(3)$ group factors (see Fig. 2 in [11]).

If kinematically allowed, the scalar diquark can also decay into a pair of squarks through the soft SUSY breaking trilinear coupling. In this case, the scalar diquark resonance can enhance the production cross section of squarks and can give some advantage to sparticle searches at the LHC.

Fermion diquarks can be pair produced through QCD processes and the production cross section is of the same order of magnitude as the production cross section of the scalar diquark pair. Since they are $R$-parity odd particles, the decay process of fermion diquarks is similar to the usual sparticle decay processes. For example, a fermion diquark can decay into squarks and quarks, and this process is similar to gluino decay, except for the difference arising from baryon number. Since the fermion diquark baryon number is twice that of the quark, it is crucial to identify its baryon number from its decay products. However, this can be quite challenging.

\section{Conclusion}

We have investigated a class of SUSY models which contain color triplet diquark fields with masses of around a few hundred $\mathrm{GeV}$ to one $\mathrm{TeV}$. The couplings between the diquark and a pair of quarks play a crucial role in observable $n-\bar{n}$ oscillation which may be tested in the near future. Based on a relatively simple model recently proposed in [9], we have investigated the collider phenomenology of the diquark fields. The phenomenological constraints require that the couplings between the diquarks and a pair of quarks should be almost flavor-blind and their magnitude should be less than unity. For the diquark couplings satisfying these constraints, we have analyzed $s$-channel scalar diquark production and its subsequent decay into the top and bottom quarks at hadron colliders. We first investigated the lower bound on the scalar diquark mass from the current observation of single top quark production at the Tevatron. We find that the diquark mass $M_{D} \gtrsim 415 \mathrm{GeV}$ for a diquark coupling constant of 0.3. At the LHC, the differential cross section of the scalar diquark production shows a resonance peak for a diquark coupling of $\mathcal{O}(0.1)$. The difference in cross sections for scalar diquark and anti-diquark at the LHC, which can be identified by comparing the number of events with top and antitop quarks, provides direct evidence of nonzero baryon number of the diquark. The scalar diquarks $D$ and $\bar{D}^{\dagger}$ are identical except for the chirality of the quarks to which they couple. Measuring the spin polarization of top quarks offers a way to distinguish between these diquarks at the LHC. 


\section{Acknowledgments}

This work is supported in part by the DOE Grant No. DE-FG02-91ER40626 (I.G., Y.M. and Q.S.) and GNSF Grant No. 07_462_4-270 (I.G.). N.O. would like to thank the Particle Theory Group of the University of Delaware for hospitality during his visit.

\section{References}

[1] See, for instance, E. Witten, Nucl. Phys. B 258, 75 (1985); G. Lazarides, C. Panagiotakopoulos and Q. Shafi, Phys. Rev. Lett. 56, 432 (1986); G. Lazarides, P. K. Mohapatra, C. Panagiotakopoulos and Q. Shafi, Nucl. Phys. B 323, 614 (1989).

[2] V. D. Angelopoulos, J. R. Ellis, H. Kowalski, D. V. Nanopoulos, N. D. Tracas and F. Zwirner, Nucl. Phys. B 292, 59 (1987); J. L. Hewett and T. G. Rizzo, Phys. Rept. 183, 193 (1989); S. F. King, S. Moretti and R. Nevzorov, Phys. Rev. D 73, 035009 (2006) arXiv:hep-ph/0510419]; J. Kang, P. Langacker and B. D. Nelson, Phys. Rev. D 77, 035003 (2008) arXiv:0708.2701 [hep-ph]].

[3] K. S. Babu and J. C. Pati, Phys. Lett. B 384, 140 (1996) arXiv:hep-ph/9606215]; M. Bastero-Gil and B. Brahmachari, Nucl. Phys. B 575, 35 (2000) arXiv:hep-ph/9907318]; J. L. Chkareuli, I. G. Gogoladze and A. B. Kobakhidze, Phys. Rev. Lett. 80, 912 (1998); J. L. Chkareuli, C. D. Froggatt, I. G. Gogoladze and A. B. Kobakhidze, Nucl. Phys. B 594, 23 (2001) arXiv:hep-ph/0003007); T. Li, D. V. Nanopoulos and J. W. Walker, arXiv:0910.0860 [hep-ph].

[4] K. S. Babu, I. Gogoladze, M. U. Rehman and Q. Shafi, Phys. Rev. D 78, 055017 (2008) arXiv:0807.3055 [hep-ph]]; S. P. Martin, arXiv:0910.2732 [hep-ph]; P. W. Graham, A. Ismail, S. Rajendran and P. Saraswat, arXiv:0910.3020 [hep-ph]; K. S. Babu, I. Gogoladze and C. Kolda, arXiv:hep-ph/0410085.

[5] T. Moroi and Y. Okada, Phys. Lett. B 295, 73 (1992); Mod. Phys. Lett. A 7, 187 (1992).

[6] S. W. Ham, S. a. Shim and S. K. OH, arXiv:1001.1129 [hep-ph].

[7] T. Ibrahim and P. Nath, arXiv:1001.0231 [hep-ph].

[8] Z. Chacko and R. N. Mohapatra, Phys. Rev. D 59, 055004 (1999) arXiv:hep-ph/9802388];

K. S. Babu and R. N. Mohapatra, Phys. Lett. B 518, 269 (2001) arXiv:hep-ph/0108089];

K. S. Babu, P. S. Bhupal Dev and R. N. Mohapatra, Phys. Rev. D 79, 015017 (2009) 
arXiv:0811.3411 [hep-ph]]; B. Dutta, Y. Mimura and R. N. Mohapatra, Phys. Rev. Lett. 96, 061801 (2006) arXiv:hep-ph/0510291.

[9] M. A. Ajaib, I. Gogoladze, Y. Mimura and Q. Shafi, Phys. Rev. D 80, 125026 (2009) arXiv:0910.1877 [hep-ph]].

[10] E. DelNobile, R. Franceschini, D. Pappadopulo and A. Strumia, Nucl. Phys. B 826, 217 (2010) arXiv:0908.1567 [hep-ph]]; T. Han, I. Lewis and T. McElmurry, arXiv:0909.2666 [hep-ph]; A. Arhrib, R. Benbrik and C. H. Chen, arXiv:0911.4875 [hep-ph].

[11] C. R. Chen, W. Klemm, V. Rentala and K. Wang, Phys. Rev. D 79, 054002 (2009) arXiv:0811.2105 [hep-ph]].

[12] H. Tanaka and I. Watanabe, Int. J. Mod. Phys. A 7, 2679 (1992).

[13] R. N. Mohapatra, N. Okada and H. B. Yu, Phys. Rev. D 77, 011701 (2008) arXiv:0709.1486 [hep-ph]].

[14] J. C. Pati and A. Salam, Phys. Rev. D 10, 275 (1974).

[15] H. Nishino et al. [Super-Kamiokande Collaboration], Phys. Rev. Lett. 102, 141801 (2009) arXiv:0903.0676 [hep-ex]].

[16] M. B. Green and J. H. Schwarz, Phys. Lett. B 149, 117 (1984); Nucl. Phys. B 255, 93 (1985); M. B. Green, J. H. Schwarz and P. C. West, Nucl. Phys. B 254, 327 (1985).

[17] T. Aaltonen et al. [CDF Collaboration], Phys. Rev. D 79, 112002 (2009) arXiv:0812.4036 [hep-ex]].

[18] S. Atag, O. Cakir and S. Sultansoy, Phys. Rev. D 59, 015008 (1999); O. Cakir and M. Sahin, Phys. Rev. D 72, 115011 (2005) arXiv:hep-ph/0508205].

[19] L. Lavoura and J.P. Silva, Phys. Rev. D 47, 2046 (1993); N. Maekawa, Phys. Rev. D 52, 1684 (1995).

[20] C. Amsler et al. [Particle Data Group], Phys. Lett. B 667, 1 (2008).

[21] F. Gabbiani and A. Masiero, Nucl. Phys. B 322, 235 (1989); J. Hagelin, S. Kelley and T. Tanaka, Nucl. Phys. B 415, 293 (1994); F. Gabbiani, E. Gabrielli, A. Masiero and L. Silvestrini, Nucl. Phys. B 477, 321 (1996) arXiv:hep-ph/9604387.

[22] C. T. Hill and S. J. Parke, Phys. Rev. D 49, 4454 (1994) arXiv:hep-ph/9312324. 
[23] K. Agashe, A. Belyaev, T. Krupovnickas, G. Perez and J. Virzi, Phys. Rev. D 77, 015003 (2008) arXiv:hep-ph/0612015; B. Lillie, L. Randall and L. T. Wang, JHEP 0709, 074 (2007) arXiv:hep-ph/0701166]; D. Choudhury and D. K. Ghosh, Int. J. Mod. Phys. A 23, 2579 (2008) arXiv:0707.2074 [hep-ph]].

[24] C. Vellidis [CDF Collaboration], arXiv:0910.3392 [hep-ex].

[25] T. Aaltonen et al. [CDF Collaboration], Phys. Rev. Lett. 101, 252001 (2008) arXiv:0809.2581 [hep-ex]]; V. M. Abazov et al. [D0 Collaboration], Phys. Rev. Lett. 103, 092001 (2009) arXiv:0903.0850 [hep-ex]]; T. Aaltonen et al. [CDF Collaboration], Phys. Rev. Lett. 103, 092002 (2009) arXiv:0903.0885 [hep-ex]].

[26] C. E. Gerber, arXiv:0909.4794 [hep-ex].

[27] H. L. Lai et al. [CTEQ Collaboration], Eur. Phys. J. C 12, 375 (2000) arXiv:hep-ph/9903282;

[28] T. Stelzer, Z. Sullivan and S. Willenbrock, Phys. Rev. D 58, 094021 (1998) arXiv:hep-ph/9807340.

[29] For studies at the LHC see, for example, F. Hubaut, E. Monnier, P. Pralavorio, K. Smolek and V. Simak, Eur. Phys. J. C 44S2, 13 (2005) arXiv:hep-ex/0508061.

[30] R. M. Harris, C. T. Hill and S. J. Parke, arXiv:hep-ph/9911288.

[31] M. Arai, N. Okada, K. Smolek and V. Simak, Phys. Rev. D 70, 115015 (2004) arXiv:hep-ph/0409273; Phys. Rev. D 75, 095008 (2007) arXiv:hep-ph/0701155; Acta Phys. Polon. B 40, 93 (2009) arXiv:0804.3740 [hep-ph]]; M. Arai, N. Okada and K. Smolek, Phys. Rev. D 79, 074019 (2009) arXiv:0902.0418 [hep-ph]]. 